\title{
On units and class numbers of pure cubic fields
}

\author{
Journal Article
}

Author(s):

Reich, Nils

Publication date:

2006

Permanent link:

https://doi.org/10.3929/ethz-b-000158014

Rights / license:

In Copyright - Non-Commercial Use Permitted

Originally published in:

Mathematika 52(1-2), https://doi.org/10.1112/S002557930000036X 


\title{
ON UNITS AND CLASS NUMBERS OF PURE CUBIC FIELDS
}

\author{
NILS REICH
}

Abstract. This is a study of relations between pure cubic fields and their normal closures. Explicit formula shows how the discriminant, regulator and class number of the normal closure can be expressed in terms of the cubic field.

In contrast to the highly developed theory of quadratic number fields, the case for the next level, cubic fields, appears different. Cubic fields (among them, the easiest case, pure cubic fields) are already very important examples for a perception which resists standard methods. A pure cubic field $k$, i.e., $k=\mathbb{Q}(\sqrt[3]{n})$, is neither abelian nor Galois, in general.

The basis of this work is the paper [2] by Dedekind. We shall extend Dedekind's results by relating the invariants of a pure cubic field $k$ to those of its normal closure $L$. In particular we show the main result

THEOREM 1. The regulator $R_{L}$ of $L$ is always either $3 R_{k}^{2}$ or $R_{k}^{2}$.

This result enables us to prove the following.

THEOREM 2. The class number $h_{L}$ of $L$ is always either $h_{k}^{2}$ or $\frac{1}{3} h_{k}^{2}$.

We will also need the following fact. It is a well-known result of Hasse [3] for arbitrary cubic fields in 1930.

Fact 1. The discriminant $d_{L}$ of $L$ satisfies $d_{L}=-3 d_{k}^{2}$.

Theorem 2 follows quite easily from Theorem 1 using the analytic class number formula and fundamental properties of Artin L-functions.

Since the discriminant of the normal closure $L$ usually is much bigger than that of the cubic field, Theorem 2 is of particular interest, because it enables us to find the class number of $L$, although the standard algorithm (see [6]) for finding the class number would be much slower in this case.

Throughout this paper $k$ will denote a pure cubic number field with conjugate fields $k^{\prime}$ and $k^{\prime \prime}$. Their normal closure will be denoted by $L$. As indicated above, we will write $d_{k}, R_{k}, h_{k}$ for the discriminant, regulator and class number of $k$.

$\S 1$. The units. In this section we will prove Theorem 1 and give a criterion for the value of $R_{L}$.

Since $L$ admits 3 pairs of complex embeddings, Dirichlet's unit theorem tells us that a system of fundamental units of $L$ consists of 2 elements. Furthermore, each of the subfields $k, k^{\prime}$ and $k^{\prime \prime}$ has only one fundamental unit. Clearly a unit of $k$ continues to be a unit in the bigger field $L$. 
Let $\mu(L)$ be the group of roots of unity of $L$. In fact, $\mu(L)=\mu_{6}$. Put

$$
E_{L}:=\mathcal{O}_{L}^{X} / \mu(L),
$$

i.e. the group of units $\mathcal{O}_{L}^{X}$ of $L$, where two units are considered equal if their ratio is a root of unity. Furthermore, let $\varepsilon$ (resp. $\mathcal{E}^{\prime}$ ) denote a fundamental unit of $k$ (resp. $k^{\prime}$ ) and define $U_{k}$ to be the subgroup of $E_{L}$ generated by $\varepsilon$ and $\varepsilon^{\prime}$.

To find out how the fundamental units of $L$ relate to those of $k$ (resp. $k^{\prime}$ ) we will determine the index $I:=\left[E_{L}: U_{k}\right]$. But first we recall a general fact.

Fact 2. For any vector $v \in \mathbb{Z}^{2}$ there exists a basis $\left\{b_{1}, b_{2}\right\}$ of $\mathbb{Z}^{2}$ such that $v=a b_{1}$, with $a \in \mathbb{N}$.

This clearly carries forward to

LEMMA 1. Let $\varepsilon$ be a fundamental unit of $k$. Then there exists a system of fundamental units $\left\{\eta_{1}, \eta_{2}\right\}$ of $L$ such that, up to a root of unity,

$$
\varepsilon=\eta_{1}^{a}, \text { for some } a \in \mathbb{N} .
$$

Using this result, we can give a bound for the index $I=\left[E_{L}: U_{k}\right]$.

THEOREM 3. The index $I=\left[E_{L}: U_{k}\right]$ is always either 1 or 3 .

Proof. Let $\varepsilon$ be a fundamental unit of $k$. Let $\varepsilon^{\prime}$ and $\varepsilon^{\prime \prime}$ be conjugates of $\varepsilon$. By Lemma 1 we can find a system $\left\{\eta_{1}, \eta_{2}\right\}$ of fundamental units in $L$, such that (up to a root of unity)

$$
\begin{gathered}
\varepsilon=\eta_{1}^{a}, \\
\varepsilon^{\prime}=\eta_{1}^{b} \eta_{2}^{c},
\end{gathered}
$$

where $a, b, c \in \mathbb{N}$, and one can easily verify that then

$$
I=a c .
$$

Since $\varepsilon \mathcal{E}^{\prime} \varepsilon^{\prime \prime}= \pm 1$, considering the relative norms of these fundamental units we find (up to a root of unity in $k^{\prime}$ ),

$$
N_{L \mid k^{\prime}}(\varepsilon)=\varepsilon \mathcal{E}^{\prime \prime}=\frac{1}{\mathcal{E}^{\prime}}=N_{L \mid k^{\prime}}\left(\eta_{1}\right)^{a} .
$$

Hence, $a=1$ and therefore $\varepsilon^{\prime}=\varepsilon^{b} \eta_{2}^{c}$.

By considering

$$
N_{L \mid k}\left(\varepsilon^{\prime}\right)=\varepsilon^{\prime} \varepsilon^{\prime \prime}=\frac{1}{\varepsilon}=\varepsilon^{2 b} N_{L \mid k}\left(\eta_{2}\right)^{c},
$$

(up to a root of unity in $k$ ) we obtain $c \mid(2 b+1)$. Similarly, we deduce from

$$
N_{L \mid k^{\prime}}\left(\varepsilon^{\prime}\right)=\varepsilon^{\prime-b} N_{L \mid k^{\prime}}\left(\eta_{2}\right)^{c}
$$


that $c \mid(2+b)$. Hence $c$ divides $2(2+b)-(2 b+1)=3$, which yields

$$
I=1 \text { or } I=3 .
$$

We immediately deduce

PROPOSITION 1. The index $I=1$ if and only if the fraction $\varepsilon^{\prime} / \varepsilon$ is not a cube in $L$ (up to a root of unity).

Proof. Using the above notation, we know that, if $I=1$, then (up to a root of unity)

$$
\frac{\varepsilon^{\prime}}{\varepsilon}=\eta_{1}^{b-1} \eta_{2},
$$

which is not a cube in $L$.

On the other hand, if $I=3$, then (up to a root of unity)

$$
\frac{\varepsilon^{\prime}}{\varepsilon}=\eta_{1}^{b-1} \eta_{2}^{3} .
$$

But, since $(2 b+1)=(b-1)+(2+b)$ and, as we have seen in the previous proof, $3 \mid(2 b+1)$ as well as $3 \mid(2+b)$, we can conclude that $b-1 \equiv 0(\bmod 3)$, from which the result follows.

Remark. Since $L$ contains a cube root of unity, to determine whether $\varepsilon / \varepsilon^{\prime}$ is a cube in $L$ one needs to examine the cubic Hilbert symbols $\left(\varepsilon / \varepsilon^{\prime},-\right)_{3, p}$ for primes $p$ of $L$ above 3 , because $\varepsilon / \varepsilon^{\prime}$ is a unit.

In order to describe the relation between the regulators we first describe the relation of the index $I=\left[E_{L}: U_{k}\right]$ to the regulators of $L$ and $k$.

Recall that $U_{k}$ denotes the subgroup of $E_{L}$ generated by the fundamental units $\varepsilon$ and $\varepsilon^{\prime}$. We will denote the regulator of $U_{k} \subset E_{L}$ by $R\left(\varepsilon, \varepsilon^{\prime}\right)$.

Note that

$$
\frac{R\left(\varepsilon, \varepsilon^{\prime}\right)}{R_{L}}=\left[E_{L}: U_{k}\right]=I,
$$

by definition. Furthermore, one can show by properties of determinants that

$$
R\left(\varepsilon, \varepsilon^{\prime}\right)=3 R_{k}^{2} .
$$

Thus, combining equations (1) and (2) we obtain that the regulators of $k$ and $L$ relate in the following way:

- if $I=1$, then $R_{L}=3 R_{k}^{2}$;

- if $I=3$, then $R_{L}=R_{k}^{2}$.

Hence, we obtain Theorem 1.

§2. The class numbers. In this section we will use Theorem 1 to prove the following proposition which is a detailed version of Theorem 2. 
PROPOSITION 2. The class numbers of $k$ and $L$ are related in the following way:

- if $I=1$, then

$$
h_{L}=\frac{1}{3} h_{k}^{2}
$$

- if $I=3$, then

$$
h_{L}=h_{k}^{2}
$$

Before we start the proof we recall the following facts (see also [5] and [7]).

- Except for the three pure cubic fields $k, k^{\prime}$ and $k^{\prime \prime}$, the only proper subfield of $L$ other than $\mathbb{Q}$ is the quadratic field $\mathbb{Q}(\omega)$, where $\omega$ represents a cubic root of unity.

- The Dedekind zeta-function corresponding to $\mathbb{Q}(\omega)$ is

$$
\zeta_{\mathbb{Q}(\omega)}(s)=\zeta(s) \mathcal{L}(L \mid \mathbb{Q}, \rho, s),
$$

where $\mathcal{L}$ denotes the Artin L-function of the alternating representation $\rho$ of the Galois group $\operatorname{Gal}(L \mid k) \cong S_{3}$. Here, $S_{3}$ means the symmetric group of order 6 and $\zeta$ denotes the Riemann zeta-function.

- The zeta-function corresponding to $k$ is

$$
\zeta_{k}(s)=\zeta(s) \mathcal{L}(L \mid \mathbb{Q}, \gamma, s),
$$

where $\gamma$ denotes the standard (i.e., 2-dimensional) representation of $S_{3}$.

To see the second and third point one can use the fact that the Dedekind zetafunction factorizes into a product of Artin L-functions (compare [6]) and then work these out explicitly.

- The analytic class number formula (see [1]) for an algebraic number field $F$ is given by

$$
\lim _{s \rightarrow 1}(s-1) \zeta_{F}(s)=\frac{2^{r_{1}+r_{2}} \pi^{r_{2}} R_{F}}{m \sqrt{\left|d_{F}\right|}} h_{F},
$$

where $m=|\mu(F)|$ is the number of roots of unity in $F$ and $r_{1}$ (resp. $r_{2}$ ) denotes the number of real (resp. complex) embeddings of $F$ into $\mathbb{C}$.

Given these facts, we can now prove Proposition 2.

Proof. Using the factorization of zeta-functions into Artin L-functions, we obtain for the residue of $\zeta_{L}$ at $s=1$

$$
\begin{aligned}
\lim _{s \rightarrow 1}(s-1) \zeta_{L}(s) & =\lim _{s \rightarrow 1}(s-1) \zeta(s) \mathcal{L}(L \mid \mathbb{Q}, \rho, s) \mathcal{L}(L \mid \mathbb{Q}, \gamma, s)^{2} \\
& =\lim _{s \rightarrow 1}(s-1) \zeta_{\mathbb{Q}(\omega)}(s) \cdot \frac{1}{(s-1)^{2} \zeta(s)^{2}} \cdot(s-1)^{2} \zeta_{k}(s)^{2} .
\end{aligned}
$$

Using the analytic class number formula it is easy to see that $\lim _{s \rightarrow 1}(s-1)$ $\zeta_{\mathbb{Q}(\omega)}(s)=\pi / 3 \sqrt{3}$ and, again by the class number formula, we can relate the 
invariants of $L$ to those of $k$ in the following way:

$$
\frac{(2 \pi)^{3} R_{L}}{6 \sqrt{\left|d_{L}\right|}} \cdot h_{L}=\frac{\pi}{3 \sqrt{3}}\left(\frac{2^{2} \pi R_{k}}{2 \sqrt{\left|d_{k}\right|}} \cdot h_{k}\right)^{2} .
$$

Hence, Proposition 2 follows immediately from inserting the results of Fact 1 and Theorem 1 into equation (4).

Since the class number is a positive integer, the following holds.

COROLlaRY 1. If $h_{k}$ is not divisible by 3 , then $I=3$ and hence $h_{L}=h_{k}^{2}$. In particular, if $h_{k}=1$ then $h_{L}=1$.

Note that for certain pure cubic fields a criterion for $h_{k}$ being divisible exactly by 3 has been given in [4].

Acknowledgements. I would like to thank Dr Richard Hill from University College London who gave me the possibility and support to accomplish the project that produced the results of this paper.

\section{References}

1. C.M. Borevich and I.R. Shafarevich. Number Theory. Academic Press (New York, 1966).

2. R. Dedekind. Über die Anzahl der Idealklassen in reinen kubischen Zahlkörpern. In Mathematische Werke, Chelsea Publishing Company (New York, 1969), 148-233.

3. H. Hasse. Arithmetische Theorie der kubischen Zahlkörper auf klassenkörpertheoretischer Grundlage. Mathematische Zeitschrift, 31 (1930), 565-582.

4. M.C. Ismaili and R. El Mesaoudi. Sur la divisibilité exacte par 3 du nombre du classes de certains corps cubiques pure. Ann. Sci. Math. Quebec, 25 (2001), 153-177.

5. J. Neukirch. Algebraische Zahlentheorie. In Ein Jahrhundert Mathematik 1890-1990, pages 587-628. Vieweg \& Sohn (Braunschweig, 1990).

6. J. Neukirch. Algebraic Number Theory. Springer-Verlag (New York, 1999).

7. J.T. Tate. Global class field theory. In Algebraic Number Theory, Academic Press (New York, 1967), 162-203.

N. Reich,

Seminar for Applied Mathematics,

ETH Zurich,

Raemistrasse 101,

8092 Zurich,

Switzerland

E-mail: nils.reich@math.ethz.ch
MSC (2000): Primary, 11R16;

Secondary, 11R29.

Received on the 2nd of June, 2005. 